\title{
Property Rights Protection of Biotechnology Innovations
}

\author{
Gokhan Ozertan, Bogazici University \\ H. Alan Love, Texas A\&M University \\ Curtis R. Taylor, Duke University \\ and \\ Diana M. Burton, Texas A\&M University \\ January 2002
}

Keywords: intellectual property, contracts, technology protection system, piracy, durable goods monopoly, biotechnology

Please address all correspondence to: H. Alan Love, Texas A\&M University, 2124 TAMU, College Station, TX 77843-2124, 979-845-3270, alove@tamu.edu

The authors wish to thank Richard Woodward, Paul Mitchell and George Davis for helpful comments. This research was supported by the Texas Agricultural Experiment Station and a grant from the US Department of Agriculture. 


\begin{abstract}
Protection of intellectual property embedded in potentially reproducible living organisms presents some unique and difficult challenges. Biological innovations like genetically modified (GM) seed are self-replicating, and as a result, represent a form of durable good with a potential product life extending far into the future. This gives rise to two problems: the need for copy protection of intellectual property contained in GM seed, and traditional durable goods price commitment problems combined with competition between new and reproduced progeny seed.

In this study, we compare contracts with use of biotechnological methods to protect intellectual property in GM seeds. Specifically, we consider three regimes: current contracts commonly used in the industry, biotechnological methods, and long-term contracts. We investigate economic welfare consequences for farmers, innovators and society of the various intellectural property protection methods using a two-period framework that permits asymmetric information and posits a probabilistic enforcement environment for pirating farmers. Results indicate that biotechnological methods yield the highest expected profit for the biotech firm. An important advantage of such a technology is that all transaction costs related with enforcement and monitoring are eliminated. However, for farmers, both current and long-term contracts are preferred to implementation of biotechnical methods. Depending on monitoring costs, long-term contracts may also be socially preferred to current contracts and biotechnical methods.
\end{abstract}




\section{INTRODUCTION}

Development of recombinant DNA techniques to move gene sequences across living organisms, in conjunction with the landmark US Supreme Court decision Diamond v. Chakrabarty (447 US 303, 1980) to make human-made microorganisms patentable, has spawned an entirely new product class of genetically modified (GM) seed. The first commercially produced GM seeds were released for sale in the US in 1996. Most of these seeds were modified to include insect or herbicide resistance. Benefits of these GM innovations are not equally valuable to all farmers, but depend on each farm's pest problems. Even so, in 1999, US farmers planted $57 \%$ of soybeans, $65 \%$ of cotton and $38 \%$ of maize production using GM seed containing these innovations (USDA, 2000). Estimates for bringing a recombinant DNA product to market range between, on average, ten years and $\$ 240$ million $(\mathrm{Ko}, 1992)$ to approximately six years and about $\$ 10$ million (Fernandez-Cornejo et al., 1999).

Protection of intellectual property (IP) embedded in potentially reproducible living organisms presents some unique and difficult challenges. Almost immediately after commercial release of GM seed, intellectual property rights protection issues arose. Biological innovations like GM seed are self-replicating, and as a result, represent a form of durable good with a potential product life extending far into the future. The patent holder of a particular GM modification becomes a durable goods monopolist. This gives rise to two problems: the need for copy protection of intellectual property contained in GM seed, and traditional durable goods price commitment problems combined with competition between new and reproduced progeny seed (Coase, 1972; Bulow, 1982; and Swan, 1980).

For some species and varieties, these problems are mitigated by natural 
variations associated with biological reproduction. The degree of variation, and often degradation in quality, from the parent depends on the species and on the degree and type of hybridization (Hansen and Knudson, 1996; Stallmann, 1986). For example, maize progeny seeds have very low production when compared to original seeds, while progeny seeds from soybeans, wheat and cotton provide yields closer to the originals. Indeed, under existing law, farmers can and often do save non-GM progeny seed to plant in the next growing cycle, only purchasing new seed when productivity falls below a critical level.

In an effort to protect intellectual property contained in patent-protected GM seed, companies have introduced legally binding contracts, Technology Use Agreements, (TUA) that assess a technology use fee and prohibit use or sale of progeny seed for planting (Monsanto, 1996-2000). However, driven by strong monetary incentives, and perhaps by a tradition of saving progeny seed for production in future periods, at least some farmers have chosen to save and use progeny seed rather than follow contract terms. This practice has come to be known as "piracy."

Piracy can significantly reduce innovator profits and firms have aggressively pursued copiers. Indeed, Monsanto has sued more than 500 farmers for planting prodeny seed in violation of TUA contracts (Pope, 1999). However, application of property rights protection to plant agriculture is both expensive and difficult. Before direct court costs of enforcement, cheaters must be identified. Farmers are geographically dispersed and tests to identify pirated GM seed are costly. Further, since plants and seed are subject to natural genetic drift, it can be hard to identify a patented plant product and define precisely when copying has occurred. This makes it difficult for patent holders of engineered 
microorganisms to prove infringement (Lesser, 1990).

Alternatives to enforcement of contracts are biotechnological solutions to intellectual property rights protection. Perhaps as a response to losses generated by piracy, biotechnology innovators have developed Technology Protection Systems (TPS) (Control of.., 1998). These systems generally work by rendering progeny GM seed sterile and thus useless to pirating farmers. Hence, TPS eliminates intellectual property enforcement issues. However, release of sterilityinducing genetic material, often refered to as "terminator technology" by its opponents, has caused environmental controversy and several environmental groups have protested its commercial introduction (Pope, 1999). Possible externalities, including environmental and ecological damages associated with introducing this technology, are discussed in Crouch (1998), McHughen (2000), and Ho et al. (1998). The result is that TPS technology has not been commercially implemented.

In this study, we compare contracts with use of TPS to protect intellectual property in GM seeds. Specifically, we consider three regimes: current TUA contracts, biological TPS solutions, and long-term contracts. We investigate economic welfare consequences for farmers, innovators and society of the various IP protection methods using a two-period framework that permits asymmetric information and posits a probabilistic enforcement environment for pirating farmers. Results indicate that TPS technology yields the highest expected profit for the biotech firm. An important advantage of such a technology is that all transaction costs related with enforcement and monitoring are eliminated. However, for farmers, both current and long-term contracts are preferred to implementation of TPS technology. Depending on monitoring costs, long-term contracts may also 
be socially preferred to current TUA contracts and TPS implementation.

\subsection{Literature}

The unlawful reproduction of patented technologies can diminish the value of patented technologies through free riding. Copying is particularly easy in computer software, print and film and recording entertainment, most literature focuses on these industries (Shy and Thisse, 1999; Banerjee, 2000; Chen and Png, 2001; Liebowitz, 1985; Novos and Waldman, 1984; Takeyama 1994, 1997; and Conner and Rumelt, 1991). Generally, results indicate piracy creates a social welfare trade-off between increased product utilization on the one hand, and underproduction of the protected good and reduced incentive to innovate on the other (Novos and Waldman, 1984). Johnson (1985) discusses two models based on Salop's (1979) product differentiation work. In his model, welfare effects of copying depend on the trade-off between the increase in consumption represented by the copies versus the reduction in demand for originals. This trade-off hinges on the supply-elasticity of products developed, and on consumers' valuation of close substitutes. Nascimento and Vanhonacker (1988) examine the firm's pricing decision for durable goods in the presence of reproduction possibilities and find that while profits are generally higher with full protection against copiers, under some conditions, partial protection might actually be more profitable. However, Liebowitz (1985) and Novos and Waldman (1984), find that in durable goods markets, copying can be viewed as a secondary market that can be used to raise firm profits by helping to facilitate price discrimination among buyer classes. The price to those most likely to facilitate pirating can be raised to partially appropriate pirating rents. 
Recently, several authors have considered piracy in products that exhibit network effects (Takeyama 1994, 1997; Conner and Rumelt, 1991; Waldman, 1993; Shy and Thisse, 1999; Banerjee, 2000). Conner and Rumelt (1991), and Takeyama (1994) find copying may benefit both patent holders and consumers if network effects are large so that consumers' valuations of the product increase with the number of other consumers who adopt the product. Shy and Thisse (1999) analyze software pirates within the context of network effects and a price-setting duopoly software industry. They find that network effects may intensify competition as rival firms race for market share. Under strong network effects, unprotected software is a noncooperative equilibrium. On the other hand, when network effects are weak, a high-price equilibrium with software protection arises. Banerjee (2000) considers software piracy in the presence of network externalities. In his single-period model, consumers, defined by their valuation of the software, are uniformly distributed on the unit interval. Contract protections and technical devices that prohibit copying are considered. Government determines monitoring and collects penalties and results hinge on government's policy choices. The welfare distribution depends on the relative costs of monitoring, presence of the anti-piracy device and other model parameters. While positive and negative network effects may play a role in biotechnology innovations, network effects are not considered in our analysis. Positive network externalities may exist in situations where a threshold usage quantity is necessary to profitably establish processing, distribution and other facilities. Negative network externalities may arise when increased adoption of a particular innovation, like insect resistance, reduces the effectiveness and profitability for all users by triggering an adaptation in insects that makes the plant even more vulnerable. 
Chen and Png (2001) find that innovator changes in price and monitoring have quantitatively different effects on potential customers. Both policies affect consumers' choice of copying and buying. While monitoring reduces copiers' expected benefits, discouraging copying, reducing price results in consumers switching from copying to buying and, at the same time, raises noncopiers' surplus. If the innovation has already been brought to market, society prefers reducing price to monitoring as a means of protecting intellectual property. Takeyama (1997) develops a two-period model that analyzes the effects of copying for a durable goods software monopoly. Copied software is not identical to the original, but has a lower quality. Takeyama concludes that Coase time-consistency (i.e., credible commitment to prices) will be problematic and that the firm may differentiate copyright and pricing policies across consumer types.

With the exception of Takeyama (1997), theoretical copyright protection literature has utilized single period models in which consumers can copy a durable good without first purchasing it. This ignores important dynamic effects associated with a durable good produced by a monopolist and is inappropriate for models that include living organisms that can only be reproduced seasonally. Assuming two types of consumers, Takeyama (1997) uses a two-period model to investigate software piracy and finds intertemporal price discrimination plays an important role in determining welfare ramifications of illegal copying. In Takeyama's model, however, copiers need not purchase the good, but can copy in both periods. Many of her results follow from the effects of network externalities.

This paper is organized as follows. In section 2 we describe our intertemporal model in a principal-agent setting. Section 3 presents the current 
TUA contract solution. In section 4, the TPS technology solution is analyzed. Section 5 describes the long-term contract solution where GM seed buyers are allowed to save progeny for use in the second period. Section 6 compares the three solutions in terms of biotech firm's profits. Farmer welfare for the three solutions is compared in section 7. Section 8 compares expected total social welfare. Conclusions are presented in section 9.

\section{AN INTERTEMPORAL MODEL WITH PIRACY}

A biotechnology firm (principal) is assumed to develop genetically modified seed for sale to farmers (agents). There are two periods, $\mathrm{t}=1,2$ in the model and $\delta$ is the discount factor, where $\delta \varepsilon[0,1]$. In period one, farmers must decide whether to buy one unit of GM seed or one unit of traditional seed. Each farmer's demand for seed is completely price inelastic. With traditional seed, farmers have the option of saving progeny seed for use in coming periods. We normalize both farmer returns from using traditional seed and the price of traditional seed to zero.

When farmers buy GM seed, they must pay a technology use fee $P_{t}$ and sign contracts (technology use agreements) with the biotech company that: a) prohibit saving progeny seed, b) institute monitoring requirements, and c) set penalties for piracy. Risk-neutral farmer agent types are defined by their potential (or inherent) benefit from using GM seed. The value of GM seed to farmer $i$ is $\theta_{i}$. Each farmer $i$ knows his $\theta_{i}$ and agents are distributed uniformly on the unit interval, $\theta \sim$ uniform $[0,1]$. Net return from using GM seed is equal to the farmers' location on the unit interval less price paid, $\theta_{i}-P_{t}$.

Once seed is bought at $\mathrm{t}=1$, the farmer has the option of pirating at $\mathrm{t}=2$. However, the copied seed may not have the same quality as the originals. Return 
from using copied seed is equal to $\alpha \theta$, where $\alpha \varepsilon[0,1]$. The parameter can also be thought of as a biological discount factor, or as biological depreciation (Lim, 1993, p.51).

The biotech firm offers GM seed priced at $P_{t}$ at the beginning of each period. The biotech firm is a risk-neutral profit maximizer. The cost of producing GM seed is normalized to be zero. While the biotech firm does not know each farmer's agent type, it does know that farmer returns are uniformly distributed along the unit interval. Thus, to catch farmers using progeny seed in violation of their TUA contracts, the firm must monitor farmers who have purchased GM seed. Monitoring is costly and we assume the biotech firm can commit to monitor against copiers. In practice, commitment might be achieved by prepaying a fixed fee for legal staff and inspectors. Monitoring activity is represented in the model by $\mu$, where $\mu$ is 0 for no monitoring and 1 for monitoring. The cost of monitoring is $C(\mu)=k \mu$, where $k>0$. When monitored, farmers are caught with probability $\vartheta \varepsilon[0,1]$. If a farmer is caught, he loses revenue from that production period and has to pay a penalty of $\lambda \varepsilon R^{+}$, which is collected by the firm.

With full information, the biotech firm could behave as a monopolist. If the biotech firm could identify which farmers would pirate in the second period, no farmer would illegally copy since he would certainly be caught. If there were no possibility of farmers reselling purchased seed, the biotech firm could also price discriminate since it would know how each farmer values GM seed. However, in this asymmetric information model, farmers know at the beginning of the game that the firm will monitor and the probability of being caught pirating. The biotech firm does not know whom will pirate, and neither 
the firm nor farmers know whom will get caught.

When the firm introduces a TPS mechanism like terminator technology into the GM seed, it controls the quality of progeny seed and can render progeny seed sterile. Hence, the farmer needs to buy GM seed each period if he wants GM seed with perfect quality. Introduction of TPS technology allows the biotech firm to behave as a nondurable goods monopolist.

\section{CURRENT CONTRACTS}

We identify four groups of farmers separated by their implicit values of GM seed. High return farmers buy GM seed in both periods, medium-return farmers buy GM seed in the first period and pirate in the second period, and low-return farmers wait to buy GM seed until the second period. The last group consists of traditional farmers who never buy GM seed and use only traditional seed. The lowest return obtained by a high-return farmer is denoted $\theta_{H}$. The lowest return obtained by a medium-return farmer is $\theta_{M}$, and the lowest return obtained by a low-return farmer is $\theta_{L}$. Traditional farmers have implicit returns from GM seed below $\theta_{L}$. This distribution is illustrated in Figure 1 .

$\begin{array}{lllll}0 & \theta_{L} & \theta_{M} & \theta_{H} & 1\end{array}$

Figure 1. Uniform Distribution of Farmer Returns from GM Seed

Time-consistency requires that the biotech firm make its profit maximizing decisions so that it has no incentive to revise its production plan. This is achieved through backward induction, i.e., by determining its optimal decisions backward in time, starting with the second period. The solution results in a subgame perfect Nash equilibrium. 


\subsection{Second Period}

A farmer has two second-period options if he purchased GM seed in the first period. A high-type farmer can either buy GM seed again, or copy seed bought in the first period. If the farmer buys in the second period, expected return will equal his location on the unit interval $\theta_{H}$ minus the price paid for GM seed $P_{2}$. If the farmer decides to copy, his expected return will equal the expected benefit from copying, $(1-\vartheta \mu) \alpha \theta_{H}$, less the expected penalty, $\vartheta \mu \lambda$. The term $(1-\vartheta \mu)$ reflects the probability of successful piracy and $\vartheta$ is the probability of being caught. To induce high-return farmers to purchase rather than pirate in the second period, the biotech firm's pricing and monitoring choices must satisfy the following incentive compatibility constraint $(I C C)$ :

$$
\theta_{H}-P_{2} \geq(1-\vartheta \mu) \alpha \theta_{H}-\vartheta \mu \lambda
$$

Assuming an interior solution at the optimum, from (1) the second period price is determined as:

$$
P_{2}=[1-(1-\vartheta \mu) \alpha] \theta_{H}+\vartheta \mu \lambda
$$

The individual rationality constraint $(I R C)$ for low-return farmers gives the necessary condition for a low-type farmer to buy GM seed:

$$
\theta_{L}-P_{2} \geq 0
$$

The left side of inequality (3) is the expected return from buying GM seed in the second period and the right side is return from using traditional seed. Combining (2) and (3), at equality we can derive the second period price as:

$$
\theta_{L}=P_{2}=[1-(1-\vartheta \mu) \alpha] \theta_{H}+\vartheta \mu \lambda
$$


The biotech firm's second period expected profit is the sum of its sales and net penalty revenues less monitoring costs:

$$
\Pi_{2}=\int_{\theta_{H}}^{1} P_{2} d \rho+\int_{\theta_{L}}^{\theta_{M}} P_{2} d \rho+\int_{\theta_{M}}^{\theta_{H}} \vartheta \mu \lambda d \rho-k \mu
$$

The first two integrals are revenue generated by selling GM seed. The last integral is revenue received as penalty from caught copiers. Equation (5) can be expressed as a function of $\theta_{H}$ and $\theta_{M}$ after substituting for $P_{2}$ :

$$
\begin{gathered}
\max _{\theta_{H}}\left[(1-(1-\vartheta \mu) \alpha) \theta_{H}+\vartheta \mu \lambda\right]\left[1-\theta_{H}+\theta_{M}-\theta_{L}\right] \\
+\vartheta \mu \lambda\left(\theta_{H}-\theta_{M}\right)-k \mu
\end{gathered}
$$

From (4), $\theta_{L}$ can be expressed as a function of $\theta_{H}$. Hence, second-period profit is a function of $\theta_{H}$ and $\theta_{M}$.

$$
\begin{array}{cc}
\max _{\theta_{H}} & \left(1-\theta_{H}\right)\left[(1-(1-\vartheta \mu) \alpha) \theta_{H}+\vartheta \mu \lambda\right] \\
& {\left[\theta_{M}-(1-(1-\vartheta \mu) \alpha) \theta_{H}-\vartheta \mu \lambda\right]} \\
& {\left[(1-(1-\vartheta \mu) \alpha) \theta_{H}-\vartheta \mu \lambda\right]+\left(\theta_{H}-\theta_{M}\right) \vartheta \mu \lambda-k \mu}
\end{array}
$$

In the second period, the biotech firm maximizes profit with respect to $P_{2}$, which can be expressed as a function of $\theta_{H}$. Solving the FOC for $\theta_{H}$ as a function of $\theta_{M}, \alpha, \vartheta, \mu$, and $\lambda$ yields the relationship between thresholds for high and medium return farmer types:

$$
\theta_{H}=\frac{1-2 \vartheta \mu \lambda+\theta_{M}}{2(2-(1-\vartheta \mu) \alpha)}
$$

After substitution into (4), $P_{2}$ becomes:

$$
\begin{aligned}
P_{2}=\theta_{L} & =\frac{1-\alpha+\alpha \vartheta \mu+2 \vartheta \mu \lambda+\theta_{M}(1-(1-\vartheta \mu) \alpha)}{2(2-(1-\vartheta \mu) \alpha)} \\
& =\frac{1+\theta_{M}-2 \theta_{H}}{2}
\end{aligned}
$$




\subsection{First Period}

For medium return farmers' incentive compatibility constraint to be satisfied, profit from purchasing GM seed in period 1 and pirating in period 2 must be at least as large as profit from purchasing traditional seed in period 1 and GM seed in period 2:

$$
\left(\theta_{M}-P_{1}\right)+\delta\left[(1-\vartheta \mu) \alpha \theta_{M}-\mu \vartheta \lambda\right] \geq \delta\left(\theta_{M}-P_{2}\right)
$$

where the left side of (10) is the discounted return from the buying-copying alternative, and the right side is the discounted return from waiting to buy until the second period. Assuming (10) holds with equality in equilibrium and using (9), the first-period price can be expressed as a function of $\theta_{M}$ and $\theta_{H}$ :

$$
P_{1}=\theta_{M}[1+\delta((1-\vartheta \mu) \alpha-1)]-\delta \vartheta \mu \lambda+\delta P_{2}
$$

In the first period the biotech firm sells GM seed to high and mediumreturn farmers. Hence, the firm's expected profit is:

$$
\Pi=\int_{\theta_{M}}^{1} P_{1} d \theta+\delta \Pi_{2}^{*}
$$

The first part of (12) is revenue generated from first period seed sales. The second part is the discounted optimal profit from optimized (7). The time-consistent subgame-perfect optimal solution is found by maximizing (12) with respect to $\theta_{M}$.

\subsection{Current Contract Solution}

We assume the biotech firm can commit to monitor so $\mu=1$. Also, for simplicity, we assume $\alpha=1$ so progeny seed are identical to orginals. Optimal 
values for $\theta_{H}^{*}, \theta_{M}^{*}, P_{2}^{*}$, and $P_{1}^{*}$ are:

$$
\begin{aligned}
& \theta_{M}^{*}=\frac{1}{2}+\frac{\delta \vartheta}{2\left(4+4 \vartheta-3 \delta \vartheta-4 \delta \vartheta^{2}\right)} \\
& \theta_{H}^{*}=\frac{3-4 \vartheta \lambda}{4(1+\vartheta)}+\frac{\delta \vartheta}{4(1+\vartheta)\left(4+4 \vartheta-3 \delta \vartheta-4 \delta \vartheta^{2}\right)} \\
& \theta_{L}^{*}=P_{2}^{*}=\frac{3 \vartheta+4 \vartheta \lambda}{4(1+\vartheta)}+\frac{\delta \vartheta^{2}}{4(1+\vartheta)\left(4+4 \vartheta-3 \delta \vartheta-4 \delta \vartheta^{2}\right)} \\
& P_{1}^{*}=\frac{2+2 \vartheta^{2}-\delta^{2} \vartheta^{2}+2 \delta^{2} \vartheta^{4}-4 \delta \vartheta^{2}-4 \delta \vartheta^{3}+4 \vartheta}{(1+\vartheta)\left(4+4 \vartheta-3 \delta \vartheta-4 \delta \vartheta^{2}\right)}-\frac{\delta \vartheta^{2} \lambda}{1+\vartheta}
\end{aligned}
$$

Profit is maximized by charging $P_{1}^{*}$ and $P_{2}^{*}$ to GM seed buyers defined by threshold values $\theta_{H}^{*}, \theta_{M}^{*}$ and $\theta_{L}^{*}$.

LEMMA 1: When $\alpha=1$ and $\mu=1$, a sufficient condition for the IRCs and ICCs to hold, and hence for $\theta_{H}^{*} \geq \theta_{M}^{*} \geq \theta_{L}^{*}$, is:

$$
1-\vartheta(1+\delta)-2 \vartheta^{2}(1-\delta \vartheta)-4 \vartheta \lambda(1+\vartheta(1-\delta \vartheta))+3 \delta \vartheta^{2} \lambda>0
$$

Lemma 1 holds when $0 \leq \vartheta \leq 0.15$ and $0 \leq \lambda \leq 1$. Proof of Lemma 1 is given in Appendix A.

Based on inspections conducted under Technology Use Agreements, Monsanto concluded that 98 percent of farmers who sign the TUAs comply with contract terms (Yeager, 1999). This implies that $\vartheta=0.02$. In addition, evidence from recent court cases suggests that the penalty typically paid by farmers caught pirating seed is equal to the technology fee for that crop (Judge Orders.., 2001). This implies a $\lambda$ value less than or equal to the price charged for GM seed. These values are consistent with parameter limitations imposed by Lemma 1. 
PROPOSITION 1: When Lemma 1 holds, $\theta_{H}^{*}$ decreases and $\theta_{L}^{*}$ increases in both $\lambda$ and $\vartheta$. Optimal $\theta_{M}^{*}$ does not depend on the penalty for piracy, $\lambda$, and rises with probability of being caught, $\vartheta . \theta_{H}^{*}, \theta_{M}^{*}$ and $\theta_{L}^{*}$ all increase in the discount factor, $\delta$.

Partial proof of Proposition 1 is given in Appendix B. Proposition 1 means that the minimum return necessary to persuade a farmer to purchase GM seed in period $1, \theta_{M}^{*}$, rises as the probability of being caught pirating in period $2, \vartheta$, rises but is independent of the penalty $\lambda$. The minimum return to high-type farmers, $\theta_{H}^{*}$, falls with increases in the probability of being caught $\vartheta$ and penalty $\lambda$, which means that these increases convince more farmers to purchase GM seed in period 2. Thus, the number of pirates, those farmers with returns between $\theta_{H}^{*}$ and $\theta_{M}^{*}$, falls as the probability of being caught and the penalty rise. The intuition is that the number of pirates falls as cost of pirating rises.

The minimum return that defines the marginal low-type farmer, $\theta_{L}^{*}$, rises with increases in the probability of being caught $\vartheta$ and penalty $\lambda$. This results follows immediately from $\theta_{L}^{*}=P_{2}^{*}$, and the biotech firm can charge a higher price for GM seed in the second period as the expected cost of piracy to the farmer rises. Equality of the defining minimum return to a low-type farmer who only buys GM seed in the second period, $\theta_{L}^{*}$, and the optimal second period price for GM seed, $P_{2}^{*}$, results because $\theta_{L}^{*}$ defines this marginal buyer and $P_{2}^{*}$ is the price that marginal farmer must pay.

Minimum returns to all farmer types rise with the discount factor $\delta$. As the discount factor rises, the implied discount rate falls. As second period returns become more important to farmers, implied by a lower discount rate, the minimum defining return to all farmer types rises. Thus, as $\theta_{H}^{*}$ rises, there are 
fewer high-type farmers who buy GM seed in both periods. As $\theta_{L}^{*}$ rises, there are more farmers who buy traditional seed in both periods. The number of farmers pirating, those with returns between $\theta_{H}^{*}$ and $\theta_{M}^{*}$, and the number buying GM seed only in the second period, those with returns between $\theta_{M}^{*}$ and $\theta_{L}^{*}$, depend on the relative magnitude of changes in these minimum defining returns $\theta_{i}^{*}$, even though all defining returns increase in discount factor $\delta$.

PROPOSITION 2: Under the current contract regime, the biotech firm charges a higher GM seed price in the first period than in the second period.

See Appendix C. In a durable goods setting, some buyers wait for lower prices in subsequent periods as they expect the monopolist to "flood the market" (Tirole, 1988, p.82).

\section{TECHNOLOGY PROTECTION SYSTEM}

When the biotech firm implements a technology protection system for GM seed, contracts are no longer necessary as seeds are no longer self-reproducing $(\alpha=0)$. There is no need for monitoring $(\mu=0)$ or penalties $(\lambda=0)$, since farmers must buy new GM seed in each period. Hence there are only two types of farmers: those who buy GM seed and those who use traditional seed. With TPS technology, farmer $i$ is willing to purchase GM seed when his net return is greater than zero:

$$
\theta-P \geq 0
$$

When the biotech firm uses TPS technology, its profit maximization reduces to a series of single period optimizations in which the biotech firm sets the optimal price for GM seed at the same level in each period. The biotech firm's profit for each period is: 


$$
\Pi=\int_{\theta}^{1} P d \theta
$$

Substituting constraint (18) into equation (19) gives:

$$
\Pi=\theta(1-\theta)
$$

and profit is maximized at:

$$
\theta^{*}=P^{*}=\frac{1}{2}
$$

PROPOSITION 3: When TPS is implemented, the biotech firm behaves as monopolist. The optimal price is equal to $\frac{1}{2}$, and $\frac{1}{2}$ of farmers purchase GM seed in each period.

Proof to Proposition 3 is given above. The biotech firm acts as a monopolist and sets price to sell to the one-half of farmers who have the highest implicit returns from GM seed.

PROPOSITION 4: When Lemma 1 holds and TPS technology is used, the price of GM seed is higher than current contract second period price, but less than first period price under the current contract regime $\left(P_{1}^{C C *} \geq P^{T *} \geq P_{2}^{C C *}\right)$. Partial proof of Proposition 4 is presented in Appendix C. With current contracts, in the first period the biotech firm charges a price higher than full monopoly price. These high price sales help to compensate for the loss from seed piracy in the second period. Additional sales can be obtained in the second period by selling at a lower than monopoly price, inducing additional low-type farmers who bought traditional seed in the first period to buy GM seed in the second period. 


\section{LONG-TERM CONTRACTS}

As an alternative to current GM seed contracts, biotech firms could write longterm (LT) contracts. A LT contract gives farmers who buy GM seed in the first period the right to plant progeny seed in the second period. With LT contracts, three types of farmers are distinguished. High-type farmers purchase GM seed in the first period and plant progeny GM seed in the second period; the lowest return to a high-type farmer is $\theta_{B}$. Middle-type farmers use traditional seed in the first period and buy GM seed for the second period only. LT contracts are offered in the first period, but seed is offered for sale in the second period. The lowest return to a middle-type farmer is $\theta_{A}$. Low-type farmers buy traditional seed in both periods. There is no need for monitoring or penalties with LT contracts, since first period buyers have the right to use the homegrown GM seed in the second period. Progeny seed are assumed identical to original seed $(\alpha=1)$.

\subsection{Second Period}

For medium-return farmers, who purchase GM seed in the second period only, the return from buying GM seed must be at least as great as using traditional seed. Hence, the individual rationality constraint is:

$$
\theta_{A}-P_{2} \geq 0
$$

At the optimum,

$$
\theta_{A}=P_{2}
$$

In the second period, the biotech firm's expected profit is:

$$
\Pi_{2}=\int_{\theta_{A}}^{\theta_{B}} P_{2} d \theta .
$$


After substitutions, equation (24) becomes:

$$
\Pi_{2}=P_{2} \theta_{B}-P_{2} \theta_{A}
$$

Biotech firm profit is maximized with respect to $P_{2}$, giving:

$$
P_{2}^{*}=\theta_{A}=\frac{\theta_{B}}{2}
$$

Combining (25) and (26), the optimal second-period profit becomes:

$$
\Pi_{2}=\frac{\theta_{B}^{2}}{4}
$$

\subsection{First Period}

For farmers who buy GM seed using LT contracts in the first period, the discounted return from two periods should be at least as great as the discounted return from using traditional seed in the first period and waiting until the second period to purchase GM seed. Hence, the incentive compatibility constraint is:

$$
\theta_{B}-P_{1}+\delta \theta_{B} \geq \delta\left(\theta_{B}-P_{2}\right)
$$

At the optimum, the first-period LT price becomes:

$$
P_{1}=\theta_{B}\left(1+\frac{\delta}{2}\right)
$$

The biotech firm's two-period objective is:

$$
\Pi=\int_{\theta_{B}}^{1} P_{1} d \rho+\delta \Pi_{2}^{*},
$$

which can be maximized with respect to first-period LT price, $P_{1}$ to give $\theta_{B}^{*}$ as a function of $\delta$ :

$$
\theta_{B}^{*}=\frac{2+\delta}{4+\delta}
$$


Substituting (31) into (26) and (29) results in optimal prices:

$$
P_{2}^{*}=\frac{\theta_{B}^{*}}{2}=\frac{2+\delta}{2(4+\delta)},
$$

and

$$
P_{1}^{*}=\theta_{B}\left(1+\frac{\delta}{2}\right)=\frac{(2+\delta)^{2}}{2(4+\delta)}
$$

Hence, the long-term contract price in period 1 is higher than in period 2 .

PROPOSITION 5: When LT contracts are available, the second-period GM price is no greater than the GM seed price when TPS technology is implemented and the first-period LT contract price is at least as large as the TPS technology GM seed price, $P_{1}^{L T *} \geq P^{T *} \geq P_{2}^{L T *}$. Further, when the biotech firm uses current contracts with monitoring and copies are identical to originals (Lemma 1 holds), it charges lower prices in both periods compared with LT contracts $P_{1}^{L T *} \geq P_{1}^{C C *}, P_{2}^{L T *} \geq P_{2}^{C C *}$.

Appendix D demonstrates Proposition 5. The LT contract solution is identical to the current contract case where seed can be perfectly copied and the biotech firm does not monitor, but assumes all farmers will pirate and sets it first period GM price to capture these rents. Since everyone who buys GM seed using a LT contract in the first period can use progeny seed, the firm would set prices accordingly. However, under current contracts, the biotech firm's optimal pricing makes allowance for the loss imposed by unauthorized copying of GM seed. This solution is analogous to the durable goods monopoly solution in a two period model. 


\section{THE BIOTECH FIRM'S EXPECTED PROFIT}

In this section, we analyze the distribution of benefits between the biotech firm and farmers across contract and TPS regimes. The biotech firm's profit is computed for each case by substituting optimal values for choice variables into total profit.

\subsection{Current Contracts}

From (12), (13) and (16), the first-period profit is:

$$
\Pi_{1}=\left(1-\theta_{M}^{*}\right) P_{1}^{*}
$$

From (5), (13), (14) and (15), the biotech firm's second-period profit is:

$$
\Pi_{2}=P_{2}^{*}\left[\left(1-\theta_{H}^{*}\right)+\left(\theta_{M}^{*}-\theta_{L}^{*}\right)\right]+\vartheta \lambda\left(\theta_{H}^{*}-\theta_{M}^{*}\right)-k
$$

where the first term is GM seed sales revenue, the second term is revenue from catching pirates, and the last term is monitoring cost. Making substitutions, the present value of profit for both periods is:

$$
\begin{array}{cc}
\Pi^{*}= & \Pi_{1}^{*}+\delta \Pi_{2}^{*}= \\
\frac{1+2 \vartheta+2 \delta \vartheta+4 \delta \vartheta \lambda+\vartheta^{2}+\delta^{2} \vartheta^{4}-2 \delta^{2} \vartheta^{3}-2 \delta \vartheta^{3}-2 \delta^{2} \vartheta^{2}+4 \delta^{2} \vartheta^{4} \lambda}{(1+\vartheta)\left(4+4 \vartheta-3 \delta \vartheta-4 \delta \vartheta^{2}\right)} \\
+\frac{4 \delta^{2} \vartheta^{4} \lambda^{2}+3 \delta^{2} \vartheta^{3} \lambda^{2}-\delta^{2} \vartheta^{3} \lambda-4 \delta \vartheta^{3} \lambda-4 \delta \vartheta^{3} \lambda^{2}-3 \delta^{2} \vartheta^{2} \lambda-4 \delta \vartheta^{2} \lambda^{2}}{(1+\vartheta)\left(4+4 \vartheta-3 \delta \vartheta-4 \delta \vartheta^{2}\right)}-\delta k .
\end{array}
$$

\subsection{TPS Technology}

From (20) and (21), profit for each period is:

$$
\Pi^{*}=P^{*}\left(1-\theta^{*}\right)=\frac{1}{4} .
$$

The present value of profit for both periods is:

$$
\Pi^{*}=\frac{1+\delta}{4} \text {. }
$$




\subsection{Long-Term Contracts}

From (30), (31) and (33), the biotech firm's first period profit is:

$$
\left\{\Pi_{1}^{*}=\left(\frac{2+\delta}{4+\delta}\right)^{2},\right.
$$

and, from (27) and (31), the biotech firm's second-period profit is:

$$
\left\{\Pi_{2}^{*}=\left(\frac{2+\delta}{2(4+\delta)}\right)^{2} .\right.
$$

Hence, the biotech firm's discounted total profit is:

$$
\Pi^{*}=\Pi_{1}{ }^{*}+\delta \Pi_{2}{ }^{*}=\frac{(2+\delta)^{2}}{4(4+\delta)} .
$$

PROPOSITION 6: The biotech firm prefers to use TPS technology. When TPS technology is not implemented, the firm prefers LT contracts to current contracts,

$\Pi^{T *} \geq \Pi^{L T *} \geq \Pi^{C C *}$. In addition, as the discount factor $\delta$ approaches zero, the biotech firm's expected LT contract profit approaches its expected profit from using TPS technology.

See Appendix E. This proposition shows that the biotech firm maximizes profit by implementing TPS technology, creating a nondurable goods monopoly. When the discount factor approaches zero, the discount rate approaches infinity and the future does not matter. In this case, the biotech firm would separately maximize expected profit in each period under the LT contract regime just as it does under a TPS technology regime.

Profits from LT contracts are greater than under current contracts because there is no monitoring cost under long-term contracts. In addition, because the biotech firm charges for two periods of GM seed use when farmers buy in the first period, it is able to capture additional rents from farmers who use 
progeny seed under the LT regime. Under current contracts, the biotech firm motivates purchase of GM seed in the second period as an alternative to pirating by lowering $P_{2}^{C C *}$. Under current contracts, the biotech firm only captures penalties from those pirating farmers that it catches; others use progeny seed without compensating the biotech firm. Even though the biotech firm charges a higher price for GM seed in the first period under current contracts, it is constrained by the cost of alternative farmer actions in the second period.

\section{FARMERS' EXPECTED WELFARE}

\subsection{Current Contracts}

Total farmer welfare is the sum of each farmer's welfare across farmer types. Farmers who do not use GM technology have economic welfare normalized to zero. Second period total farmer welfare for the current contract regime for high-, medium- and low-type farmers is:

$$
\begin{aligned}
W_{2}^{*}= & \int_{\theta_{H}^{*}}^{1}\left(\rho-P_{2}^{*}\right) d \rho+\int_{\theta_{L}^{*}}^{\theta_{M}^{*}}\left(\rho-P_{2}^{*}\right) d \rho+\int_{\theta_{M}^{*}}^{\theta_{H}^{*}}((1-\vartheta) \rho-\vartheta \lambda) d \rho, \\
W_{2}^{*}= & \frac{1}{2}-\theta_{L}^{*}+\theta_{L}^{*} \theta_{H}^{*}-\theta_{L}^{*} \theta_{M}^{*} \\
& +\frac{\left(\theta_{L}^{*}\right)^{2}}{2}-\frac{\vartheta\left(\theta_{H}^{*}\right)^{2}}{2}-\vartheta \lambda \theta_{H}^{*}+\vartheta \frac{\left(\theta_{M}^{*}\right)^{2}}{2}+\vartheta \lambda \theta_{M}^{*} .
\end{aligned}
$$

In the first period, farmers' expected welfare is:

$$
\begin{aligned}
& W_{1}^{*}=\int_{\theta_{M}^{*}}^{1}\left(\rho-P_{1}^{*}\right) d \rho, \\
& W_{1}^{*}=\frac{1}{2}-P_{1}^{*}-\frac{\left(\theta_{M}^{*}\right)^{2}}{2}+P_{1}^{*} \theta_{M}^{*}
\end{aligned}
$$


Hence, the present value of total farmer welfare is:

$$
\begin{array}{cc}
W^{*}= & W_{1}^{*}+\delta W_{2}^{*}=\frac{1+\delta}{2}-\theta_{L}^{*}+\theta_{L}^{*}\left(\theta_{H}^{*}-\theta_{M}^{*}\right) \\
& -\vartheta \lambda\left(\theta_{H}^{*}-\theta_{M}^{*}\right)-\delta P_{1}^{*}\left(1-\theta_{M}^{*}\right)-\frac{(\delta-\vartheta) \theta_{M}^{* 2}}{2}+\frac{\theta_{L}^{* 2}}{2}-\frac{\vartheta \theta_{H}^{* 2}}{2}
\end{array}
$$

\subsection{TPS Technology}

Under the TPS technology regime, farmers' welfare is:

$$
W^{*}=\int_{\frac{1}{2}}^{1}\left(\rho-P^{*}\right) d \rho=\frac{1}{8}
$$

for each period. If we consider two periods with discount factor, $\delta$, total farmer welfare is:

$$
W^{*}=W_{1}^{*}+\delta W_{2}^{*}=\frac{1+\delta}{8}
$$

\subsection{Long-Term Contracts}

For the LT contract case, expected welfare for each farmer type is:

$$
\begin{aligned}
& W_{B}^{*}=\int_{\theta_{B^{*}}}^{1}\left[(1+\delta) \rho-P_{1}^{*}\right] d \rho=\frac{1+\delta}{2}-\frac{(2+\delta)^{2}(3+\delta)}{2(4+\delta)^{2}} \\
& W_{A}^{*}=\underset{\substack{\theta_{B^{*}} \\
\theta_{A}^{*} \int}}{\left(\rho-P_{2}^{*}\right) d \rho=\frac{(2+\delta)^{2}}{8(4+\delta)^{2}}}
\end{aligned}
$$

Total welfare for LT contract is:

$$
W^{*}=W_{B}^{*}+\delta W_{A}^{*}=\frac{1+\delta}{2}-\frac{3(2+\delta)^{2}}{8(4+\delta)}
$$




\subsection{Comparison of Farmers' Expected Welfare}

When farmers' expected welfare for the three mechanisms are compared, the following proposition is obtained:

PROPOSITION 7: TPS technology results in lower expected farmers' welfare compared with both the current contract and LT contract regimes. Current contracts result in higher expected welfare than LT contracts.

See Proposition 7 in Appendix F.

From the farmers' point of view, use of TPS technology results in lower expected welfare compared to current and LT contracts. With TPS technology, the biotech firm charges the monopoly price in each period and gets maximum economic rents from farmers in every period. When TPS technology is not implemented, current contracts provide higher expected farmer welfare, with at least some farmers benefiting from pirating. Further, under either current or LT contract regimes, farmers benefit from a lower second period price than in the monopoly TPS case.

With LT contracts, the biotech firm optimally adjusts its pricing policy to accommodate farmers planting progeny seed in the second period. Under the current contract regime, the possibility of piracy imposes an additional pricing constraint on the biotech firm. In addition, farmer welfare directly benefits from successful piracy.

\section{SOCIAL WELFARE}

Total welfare is the sum of the biotech firm's expected profit and farmers' expected welfare. 
PROPOSITION 8: Implementing TPS technology results in lower expected total welfare compared to LT contracts. If monitoring cost, $\mathrm{k}$, is greater than 0.097, LT contracts always result in greater expected total welfare compared to current contracts. If $\mathrm{k}$ is less than 0.009 , expected total welfare with current contracts is always greater than expected total welfare with TPS technology.

See Appendix G. While TPS technology results in highest biotech firm (monopoly) profit, farmer welfare and total welfare are lower. Under both the current contract and long-term contract regimes, farmers benefit from GM seed durability.

In this model, under current contracts, monitoring cost is fixed and does not affect the probability of catching pirates nor the penalty. Hence, monitoring cost simply reduces economic welfare by reducing biotech firm profit in the second period. If monitoring cost is above a critical level, total welfare of the current contract regime falls below that of long-term contracts. If monitoring cost is below a critical level, total economic welfare obtainable from the current contract regime is greater than that from a TPS regime. However, since the biotech firm has lower expected profit under both contract regimes when compared to the TPS case, its investment in research and development for future products might be lessened, reducing social welfare in the long-run.

\section{CONCLUSION}

We analyze two contract mechanisms and a biotechnological protection device for intellectual property contained in biologically reproducible agricultural crops. A biotech firm sells GM seed to farmers in a two-period model framework with asymmetric information where the biotech firm can not identify the innovation's value to each farmer. 
From the biotech firm's perspective, TPS technology clearly results in highest expected profit, as this regime results in monopoly profit. An important advantage to the firm of this technology is that enforcement and monitoring costs are eliminated. Long-term contracts provide the next highest profit level, with current contracts providing least profit. From the farmer's perspective, both current and LT contracts are preferred to the TPS regime, since these contracts result in higher expected welfare. Farmers who pirate prefer current contracts, as they realize benefits from GM seed without paying for it. Depending on monitoring cost, LT contracts are preferred to current contracts in terms of expected total welfare. If monitoring cost is high, it sufficiently depresses biotech firm profit and reduces total welfare. Alternatively, if the number of successful pirates is large and cost of monitoring is small, then total welfare is maximized under the current contract regime.

Our results indicate that LT contracts offer a viable alternative to TPS technology. LT contracts protect intellectual property investments more effectively than do current contracts. Given these results, the question arises as to why long-term contracts have not been adopted by the industry. One answer might be that the long-term contract price would be sufficiently high to discourage farmers from adopting unproven GM seed technology. Now that GM seed has been widely adopted and farmers know its value, we expect to see contracts evolve into longer term instruments.

Interpretation of model results should be done cautiously. First, this is a stylized model and it may be difficult to generalize all results. Second, when LT contracts are signed, no monitoring costs are incurred as contracts run the entire analytical horizon. In a multi-perioid setting, if contract length were less than 
the time horizon, then monitoring cost would be incurred after the end of the contract period. Also, this model ignores the possibility of commercial piracy, where farmers sell GM seed progeny for profit.

Further research might consider the effects of copy quality and continuous monitoring on the optimal contract mechanism. Another extension might be inclusion of commercial piracy. Commercial piracy represents a significant roadblock to introducing GM seed in developing countries.

\section{REFERENCES}

Banerjee, D., 2000, "Software Piracy: A Strategic Analysis and Policy Instruments," Working Paper, Bond University.

Bulow, J., 1982, "Durable Goods Monopolists," Journal of Political Economy, 90, 314-332.

Chen, Y. and I. Png, 2001, "Software Pricing and Copyright: Enforcement Against End-Users," Working Paper, National University of Singapore, Available on-line at: http://www.comp.nus.edu.sg/ipng.

Coase, R., 1972, "Durability and Monopoly," Journal of Law and Economics, $15,143-149$.

Conner, K. and R. Rumelt, 1991, "Software Piracy: An Analysis of Protection Strategies," Management Science, 37, 125-139. 
"Control of Plant Gene Expression," U.S. Patent and Trademark Office. Patent No: 5,723,765. March 3, 1998.

Crouch, M., 1998, "How the Terminator Terminates: An Explanation for the Non-Scientist of a Remarkable Patent for Killing Second Generation Seeds of Crop Plants," Available on-line at: http://www.bio.indiana.edu/people/terminator.html.

Fernandez-Cornejo, J., C. Klotz-Ingram and S. Jans, 1999, "Farm-Level Effects of Adopting Genetically Engineered Crops in the U.S.A," Available on-line at: http://agecon.lib.umn.edu, June 1999.

Hansen, L. and M. Knudson, 1996, "Property Right Protection of Reproducible Genetic Material," Review of Agricultural Economics, 18, 403-414.

Ho, M., H. Meyer and J. Cummins, 1998, "The Biotechnology Bubble," The Ecologist, 28, 146-153.

Johnson, W. R., 1985, ”The Economics of Copying,” Journal of Political Economy, 93, p. 158-174.

"Judge Orders Canola Farmer to Pay Monsanto 20,000 for Patent Infringement," 2001, Agence France Press. Available on-line at: http://www.biotechinfo.net/judge_orders.html, May 24, 2001.

Ko, Y., 1992, "An Economic Analysis of Biotechnology Patent Protection," Yale 
Law Journal, 102, 777-804.

Lesser, W., 1990, "Sector Issues II: Seeds and Plants," in Strengthening Protection of Intellectual Property in Developing Countries: A Survey of the Literature. Washington, DC, World Bank, Discussion Papers 112.

Liebowitz, S., 1985, "Copying and Indirect Appropriability: Photocopying of Journals," Journal of Political Economy, 93, 945-957.

Lim, P., 1993, "The Privatization of Species: An Economic History of Biotechnology and Intellectual Property Rights in Living Organisms," Ph.D. dissertation, Stanford, CA: Stanford University.

McHughen, A., 2000, "Biotechnology and Food, Second Edition," American Council on Science and Health, Available on-line at: http://www.acsh.org.

Monsanto, 1996-2000. Technology Use Agreement, St.Louis, MO.

Nascimento, F. and W. Vanhonacker, 1988, "Optimal Strategic Pricing of Reproducible Consumer Products," Management Science, 34, 921-937.

Novos, I. and M. Waldman, 1984, "The Effects of Increased Copyright Protection: An Analytic Approach,” Journal of Political Economy, 92, 236-246.

Pope, C., 1999, "The Bad Seeds: How the Biotechnology Industry Packages 
Nature for Profit," Sierra, 84, May/June 1999, 18-19.

Salop, S., 1979, "Monopolistic Competition with Outside Goods," Bell Journal of Economics, 10, 141-156.

Shy, O. and J. Thisse, 1999, "A Strategic Approach to Software Protection," Journal of Economics and Management Strategy, 8, 163-190.

Stallmann, J.I., 1986, "Impacts of the 1930 Plant Patent Act on Private Fruit Breeding Investment," Ph.D. Dissertation, East Lansing: Michigan State University, 1986.

Swan, P., 1980, 'Alcoa: The Influence of Recycling on Monopoly Power,' Journal of Political Economy, 88, 76-90.

Takeyama, L., 1994, "The Welfare Implications of Unauthorized Reproduction of Intellectual Property in the Presence of Demand Network Externalities," Journal of Industrial Economics, 42, 155-166.

Takeyama, L., 1997, "The Intertemporal Consequences of Unauthorized Reproduction of Intellectual Property," Journal of Law and Economics, 40, 511-522.

Tirole, J., 1988, The Theory of Industrial Organization, Cambridge, MA: MIT Press. 
U.S. Department of Agriculture, 2000, Production Practices for Major Crops in US Agriculture, 1990-1997. ERS:Statistical Bulletin No. 969, August 2000.

Waldman, M., 1993, "A New Perspective on Planned Obsolescence," Quarterly Journal of Economics, 108, 273-283.

Yeager, P., 1999, "Monsanto Contracts Demand Too Much," The Western Producer, Available on-line at: http://www.producer.com, April 15, 1999.

\section{APPENDIX A. LEMMA 1}

For our model to be valid the following two conditions must be satisfied:

I: Minimum return to a high type farmer should be greater than the minimum return to a medium-type farmer:

$$
\theta_{H}^{*} \geq \theta_{M}^{*}
$$

Subtracting equation (13) $\theta_{M}^{*}$ from (14) $\theta_{H}^{*}$ and multiplying through by the (positive) denominator yields (17).

II: Minimum return to a medium-type farmer should be greater than minimum return to a low-type farmer:

$$
\theta_{M}^{*} \geq \theta_{L}^{*}
$$

Subtracting (15) $\theta_{L}^{*}$ from (13) $\theta_{M}^{*}$ and multiplying through by the (positive) denominator gives

$$
2+\vartheta(1-\vartheta-\delta-\delta \vartheta(1-\vartheta))-4 \vartheta \lambda\left(1+\vartheta-\delta \vartheta^{2}\right)+3 \delta \vartheta^{2} \lambda>0
$$


This condition is less restrictive than (17), so if equation (17) holds within the domains of $\vartheta$ and $\delta$, regardless of $\lambda$, then equation (54) will hold.

$$
\begin{array}{cc}
\frac{d(.)}{d \delta}= & -\vartheta+2 \vartheta^{3}+4 \vartheta^{3} \lambda \\
& +3 \vartheta^{2} \lambda=-\vartheta(1-\vartheta(2 \vartheta+4 \vartheta \lambda+3 \lambda))<0 \\
\frac{d(.)}{d \vartheta}= & -1-\delta-4(\vartheta+\lambda+2 \vartheta \lambda)+6 \delta \vartheta(\vartheta+\lambda+2 \vartheta \lambda) \\
= & -1-\delta-2(\vartheta+\lambda+2 \vartheta \lambda)(2-3 \delta \vartheta)<0 \\
\frac{d(.)}{d \lambda}= & -4 \vartheta-4 \vartheta^{2}+4 \vartheta^{3} \delta+3 \vartheta^{2} \delta \\
= & -4 \vartheta\left(1-\vartheta^{2} \delta\right)-4 \vartheta^{2}\left(1-\frac{3}{4} \delta\right)<0
\end{array}
$$

When evaluated at maximal parameter values $(\vartheta=0.15, \lambda=1, \delta=1)$, Lemma 1 takes the value 0.05275 . Since (17) is monotonically decreasing in model parameters, any parameter value smaller than its upper limit will result in a larger positive value for condition (17). This proves Lemma 1.

We further investigate Lemma 1 with the following numeric analysis. Let $\delta$ and $\lambda$ take 10 values from 0.1 to 1 , increasing by 0.1 . Let $\vartheta$ take 10 values within the interval $[0.015,0.15]$, increasing by 0.015 . This results in 1000 combinations of the three parameters. Equation (17) is calculated for each combination as well as each $\theta^{*}$. These simulations result in the following minimum and maximum values, where all 1000 parameter combinations satisfy Lemma 1:

$\begin{array}{ccccc} & \text { Lemma1(17) } & \theta_{H}^{*} & \theta_{M}^{*} & \theta_{L}^{*} \\ \text { Max } & 0.97697 & 0.73836 & 0.51847 & 0.22947 \\ \text { Min } & 0.05275 & 0.52246 & 0.50018 & 0.01256\end{array}$




\section{APPENDIX B. PROPOSITIONS 1 AND 2}

The comparative statics for the optimal values of $\theta_{H}^{*}, \theta_{M}^{*}$ and $\theta_{L}^{*}$ are:

$$
\begin{aligned}
& \frac{d \theta_{H}^{*}}{d \lambda}=\frac{-4 \lambda}{4(1+\vartheta)}<0 \\
& \frac{d \theta_{M}^{*}}{d \lambda}=0 \\
& \frac{d \theta_{L}^{*}}{d \lambda}=\frac{4 \vartheta}{4(1+\vartheta)}>0 \\
& \frac{d \theta_{H}^{*}}{d \delta}=\frac{\vartheta}{\left(4+4 \vartheta-3 \delta \vartheta-4 \delta \vartheta^{2}\right)^{2}}>0 \\
& \frac{d \theta_{M}^{*}}{d \delta}=\frac{2 \vartheta(1+\vartheta)}{\left(4+4 \vartheta-3 \delta \vartheta-4 \delta \vartheta^{2}\right)^{2}}>0 \\
& \frac{d \theta_{L}^{*}}{d \vartheta}= \\
& \frac{d \theta_{L}^{*}}{d \vartheta}=\frac{2 \delta\left(\delta \vartheta^{2}+1\right)}{\left(4+4 \vartheta-3 \delta \vartheta-4 \delta \vartheta^{2}\right)^{2}}>0 \\
& \frac{d \theta_{H}^{*}}{d \vartheta} ? 0
\end{aligned}
$$

Two expressions are not signable, so numeric values for $d \theta_{H}^{*} / d \vartheta, d \theta_{L}^{*} / d \vartheta$ are used. In addition, $P_{1}^{*}, P_{2}^{*}$, and $\left(P_{1}^{*}-P_{2}^{*}\right)$ are calculated using the simulation 
described in Lemma 1.

$$
\begin{array}{cccccc} 
& d \theta_{H}^{*} / d \vartheta & d \theta_{L}^{*} / d \vartheta & P_{1}^{C C *} & P_{2}^{C C *} & P_{1}^{C C *}-P_{2}^{C C *} \\
\text { Max } & -12.69587 & 36.20004 & 0.53778 & 0.22947 & 0.49284 \\
\text { Min } & -36.06749 & 13.72633 & 0.50052 & 0.01256 & 0.27359 \\
\text { CC: Current contracts } & & &
\end{array}
$$

It is easy to see the signs of the comparative statics from the minimums and the maximums. For each parameter combination, the difference between the first and the second period prices for current contracts is always positive.

\section{APPENDIX C. PROPOSITION 4}

It can be shown that $\left(P^{T *}-P_{2}^{C C *}\right)$ is decreasing in all three parameters:

$$
\begin{aligned}
& \frac{d\left(P^{T *}-P_{2}^{C C *}\right)}{d \vartheta}=-\frac{12+16 \lambda+6 \delta^{2} \vartheta^{2}-24 \vartheta^{3} \delta-40 \delta \vartheta^{2}+13 \vartheta^{4} \delta^{2}-16 \delta \vartheta+32 \vartheta \lambda+24 \vartheta}{\left(4 \delta \vartheta^{2}+3 \delta \vartheta-4 \vartheta-4\right)^{2}(1+\vartheta)^{2}} \\
-\frac{9 \lambda \delta^{2} \vartheta^{2}-56 \vartheta^{2} \lambda \delta-32 \vartheta^{3} \lambda \delta+24 \lambda \delta^{2} \vartheta^{3}+12 \vartheta^{2}+16 \lambda \delta^{2} \vartheta^{4}-24 \lambda \delta \vartheta+18 \vartheta^{3} \delta^{2}+16 \vartheta^{2} \lambda}{\left(4 \delta \vartheta^{2}+3 \delta \vartheta-4 \vartheta-4\right)^{2}(1+\vartheta)^{2}}<0 & \\
\frac{d\left(P^{T *}-P_{2}^{C C *}\right)}{d \delta} & =-\frac{\vartheta^{2}}{\left(4 \delta \vartheta^{2}+3 \delta \vartheta-4 \vartheta-4\right)^{2}}<0 \\
\frac{d\left(P^{T *}-P_{2}^{C C *}\right)}{d \lambda} & =-\frac{\vartheta}{1+\vartheta}<0 .
\end{aligned}
$$

When evaluated at maximal simulation parameter values $(\vartheta=0.15, \lambda=1, \delta=$ $1),\left(P^{T *}-P_{2}^{C C *}\right)$ takes the value 0.27053 . This implies that for any other parameter combination within their respective domains the difference between two prices will be a larger positive number.

We cannot provide a similar demonstration for the difference $\left(P^{T *}-\right.$ $\left.P_{1}^{C C *}\right)$, since derivatives result in alternating signs. Hence, for this case, a 
numerical simulation is constructed based on the simulation of Lemma 1 values described in Appendix A.

$$
\begin{array}{ccc} 
& P^{T *}-P_{1}^{C C *} & P^{T *}-P_{2}^{C C *} \\
\text { Max } & -0.00052 & 0.48744 \\
\text { Min } & -0.03778 & 0.27053
\end{array}
$$

CC: Current contracts; T: TPS

Calculated values show that price in the TPS case is always less than first period current contract price but greater than second period current contract price.

\section{APPENDIX D. PROPOSITION 5}

For the comparison of prices between LT contracts and TPS, it is obvious that in the first period, for $0 \leq \delta \leq 1$, the long-term contract price is greater than the first period price with TPS, $P_{1}^{L T *}>P^{T *}$ :

$$
\frac{(2+\delta)^{2}}{2(4+\delta)}>\frac{1}{2}
$$

In the second period, $P^{T *}>P_{2}^{L T *}$ :

$$
\frac{2+\delta}{2(4+\delta)}<\frac{1}{2}
$$

which proves the first part of Proposition 5.

For the differences between LT contract prices and current contract prices, the differences are not signable within parameter domains. Derivatives of the differences are not monotonic in the model parameters. Hence, numerical simulations are provided for this part of the proposition.

$$
\begin{array}{ccccc} 
& P_{1}^{L T *} & P_{2}^{L T *} & P_{1}^{C C *} & P_{2}^{C C *} \\
\text { Max } & 0.90000 & 0.30000 & 0.53778 & 0.22947 \\
\text { Min } & 0.53780 & 0.25610 & 0.50052 & 0.01256 \\
\text { CC: current contracts, LT: LT contracts }
\end{array}
$$


LT contract prices are higher than current contract prices in both periods.

\section{APPENDIX E. PROPOSITION 6}

From (38) and (41) for LT contracts and TPS technology, it is easy to show that, for all $\delta>0, P i^{T *}>P i^{C C *}$

$$
\frac{1+\delta}{4}>\frac{(2+\delta)^{2}}{4(4+\delta)}
$$

Remaining results are shown through numeric simulations as described above.

$$
\begin{aligned}
& \Pi^{C C *} \quad \Pi^{T *} \quad \Pi^{L T *} \\
& \begin{array}{llll}
\text { Max } & 0.42639 & 0.50000 & 0.45000
\end{array} \\
& \begin{array}{llll}
\text { Min } & 0.25116 & 0.27500 & 0.26890
\end{array} \\
& \text { CC: current contract, T: TPS, LT: LT contract } \\
& \Pi^{T *}-\Pi^{C C *} \Pi^{T *}-\Pi^{L T *} \Pi^{L T *}-\Pi^{C C *} \\
& \begin{array}{llll}
\text { Max } & 0.23844 & 0.05000 & 0.18844
\end{array} \\
& \begin{array}{llll}
\text { Min } & 0.00739 & 0.00610 & 0.00129
\end{array}
\end{aligned}
$$

CC: current contract, T: TPS, LT: LT contract

In the numerical example, cost of monitoring is not included in expected profit calculation for current contracts. Including the (negative) cost component will make the current contract profit values smaller. However, even without this component, current contract profit values are smaller than both LT contract and TPS profit values. Hence, omission of the cost component does not affect our results.

\section{APPENDIX F. PROPOSITION 7}

From (48) and (51) for LT contracts and TPS technology, it can be easily shown that for all $\delta>0$, farmer welfare for long-term contracts is greater than under 
TPS technology, $W^{T *}>W^{L T *}$ :

$$
\frac{1+\delta}{2}-\frac{3(2+\delta)^{2}}{8(4+\delta)}>\frac{1+\delta}{8}
$$

Remaining results are demonstrated through numeric simulations as described above.

$$
\begin{array}{cccc} 
& W^{C C *} & W^{T *} & W^{L T *} \\
\text { Max } & 0.61022 & 0.25000 & 0.32500 \\
\text { Min } & 0.15379 & 0.13750 & 0.14665
\end{array}
$$

CC: current contract, T: TPS, LT: LT contract

$$
\begin{array}{cccc} 
& W^{C C *}-W^{L T *} & W^{C C *}-W^{T *} & W^{L T *}-W^{T *} \\
\operatorname{Max} & 0.28522 & 0.36022 & 0.07500 \\
\text { Min } & 0.00715 & 0.01629 & 0.00915 \\
\text { CC: current contract, T: TPS, LT: LT contract }
\end{array}
$$

Expected farmer welfare under either current or LT contracts is greater than expected welfare with TPS technology. Current contracts result in higher expected farmer welfare compared with LT contracts.

\section{APPENDIX G. PROPOSITION 8}

From (38), (41), (48) and (51) for LT contracts and TPS, it can be shown that for all $\delta>0$, total welfare under long-term contracts is greater than total welfare under TPS technology, $S W^{L T *}>S W^{T *}$ :

$$
\frac{1+\delta}{2}-\frac{3(2+\delta)^{2}}{8(4+\delta)}+\frac{(2+\delta)^{2}}{4(4+\delta)}>\frac{3(1+\delta)}{8}
$$

Remaining results are demonstrated numerically as before:

$$
\begin{array}{cccc} 
& S W^{C C *} & S W^{T *} & S W^{L T *} \\
\text { Max } & 0.87179 & 0.75000 & 0.77500 \\
\text { Min } & 0.42141 & 0.41250 & 0.41555
\end{array}
$$




$\begin{array}{cccc} & S W^{C C *}-S W^{L T *} & S W^{C C *}-S W^{T *} & S W^{L T *}-S W^{T *} \\ \operatorname{Max} & 0.09679 & 0.12179 & 0.02500 \\ \operatorname{Min} & 0.00586 & 0.00891 & 0.00305\end{array}$

CC: current contract, T: TPS, LT: LT contract

Expected total social welfare is the sum of expected firm profit and expected farmer welfare less monitoring cost. Numbers stated in the table above do not include monitoring cost. As long as cost of monitoring (k) is less than 0.009, current contracts result in higher expected social welfare than TPS technology. Expected social welfare with LT contracts is greater than with TPS technology. If $\mathrm{k}$ is greater than 0.097 , LT contracts also result in higher expected social welfare than current contracts. 